\title{
Towards a fuzzy-linguistic based social network sentiment-expression system
}

\author{
Valerio Perticone $^{1}$ Francesco D'Aleo $^{1}$ Giovanni Rizzo $^{1}$ Marco Elio Tabacchi $^{1,2}$ \\ ${ }^{1}$ DMI, Università degli Studi di Palermo \\ ${ }^{2}$ Istituto Nazionale di Ricerche Demopolis, Italy
}

\begin{abstract}
Liking allows users of Social Networks, blogs and online magazines to express their support of posts and artifacts by a simple click. Such function is very popular but lacks semantic power, and some platforms have augmented it by allowing to choose a pictographic depiction corresponding to a feeling. What is gained in depth is lost in simplicity, and the wide acceptance liking has enjoyed did not carried to the sentiment version. We outline a sentiment-expression hybrid system based on textual analysis and linguistic fuzzy Markov chains overcoming the intrinsic limitations of liking without burdening the user with complex choices.
\end{abstract}

Keywords: social networks, sentiment analysis, linguistic fuzzy Markov chains

\section{Introduction}

A common practice in the daily usage of Social Networks (SN) [1] is the so-called liking [2]; users can express their support for content posted by other users be it an image, post, short text, video or any other multimedia artifact - by pressing a specific button, usually placed under the aforementioned content. The naming is due to Facebook using the term like to label the button, but under different nomenclatures (e.g. +1 for Google Plus) the same function exists in almost any currently used SN. Liking enjoys an immense popularity, and can certainly be pinpointed as a fundamental feature of a truly social internet context [2]. Among the reasons for such favour, the extreme lightness of the cognitive load of the act of liking itself has to be recognized: liking is conceptually an atomic operation, very simple to carry and with almost no time or resources cost associated. As a further confirmation, SN developers have carefully studied the visual details (colour, position, dimension) of liking buttons, as an incentive to their use, and especially so on mobile platforms, where space is at a premium (see Figure 1).

The extreme simplicity of the act of liking is one of the reasons of its success, but also a limiting factor that has been the focus of criticism and debate. In the present form liking lacks a semantic level - as no specific feeling apart from a general sense of support is expressed. This situation has been exacerbated by SN's association of intrinsically positive terms with the act of liking, up to the paradoxical situation of expressing linguistic pleasure for other people's misfortunes in order to support them.

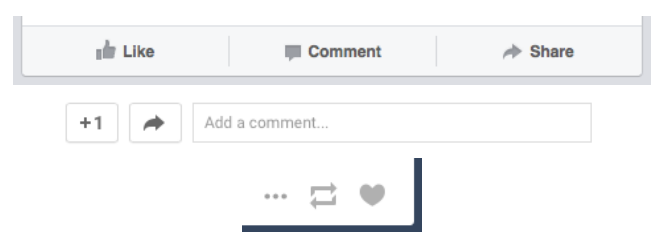

Figure 1: Representation of liking buttons in popular $\mathrm{SN}$, on desktop and mobile clients. From top to bottom Facebook, Google Plus, Tumblr.

In December 2014, in response to a question regarding the introduction of a Dislike button, Mark Zuckerberg, $\mathrm{CEO}$ and founder of Facebook Inc., argued that «A lot of times people share things on Facebook that are sad moments in their lives. Often people tell us that they don't feel comfortable pressing "like" because "like" isn't the appropriate sentiment.».[3]

\subsection{From liking to sentiment expression}

A number of blogging platform and SN have extended the concept of liking in an effort to overcome such limitations and extract more semantic information from users' interaction with the system. Especially in contexts where language may constitute a barrier such systems employ a pictographic description of the feelings associated with the elicited emotion. Such feelings are often represented through symbols from emoji, an agreed-upon set of pictograms representing a number of concrete and abstract concepts. Emoji became available in 1999 on Japanese mobile phones, and, according to the Unicode Consortium, "represent things such as faces, weather, vehicles and buildings, food and drink, animals and plants, or icons that represent emotions, feelings, or activities". In 2007 Google completed the conversion of "enhanced emoticons" to Unicode privateuse codes, and in 2009 a set of 722 Unicode characters was defined as the union of Japanese emoji characters. More pictographs were added in 2010, 2012 and 2014. [4] In November 2013, a survey revealed that 74 percent of people in the U.S. and 82 percent in China stated that they have used pictographs or cartoonish images in non-verbal communications with their relatives or friends while on SN. [5] This suggests that emoji can be usefully employed to express feelings or emotions in absence of elements such facial expressions or voice cues. [6] The appearance of emoji can vary significantly in shape, and emoji do not have to look the same on all devices: for a given emoji, any pictorial representation 


\begin{tabular}{|c|c|c|c|}
\hline & BW & Apple & Google \\
\hline $\mathrm{a}$ & $\Theta$ & $\Theta$ & $\Theta$ \\
$\mathrm{b}$ & $\Theta$ & $\ddots$ & $\ddots$ \\
$\mathrm{c}$ & $\ddots$ & $\ddots$ & $\approx$ \\
$\mathrm{d}$ & $\ddots$ & $\ddots$ & $\ddots$ \\
$\mathrm{e}$ & $\ddots$ & $\ddots$ & $\ddots$ \\
$\mathrm{f}$ & $\ddots$ & $\ddots$ & $\because$ \\
\hline
\end{tabular}

Table 1: Representation of feelings through emoji pictograms from different character sets.

\begin{tabular}{|c|c|c|c|}
\hline & Description & Code & Feeling \\
\hline a & face with tears of joy & $1 F 602$ & Happiness \\
b & unamused & $1 F 612$ & Disgust \\
c & angry & $1 F 620$ & Anger \\
d & crying face & $1 F 622$ & Sadness \\
e & fearful & $1 F 628$ & Fear \\
f & face with open mouth & $1 F 62 E$ & Surprise \\
\hline
\end{tabular}

Table 2: Additional data regarding the pictographic representation of six basic human emotions.

based on both the name or the representative glyph is considered an acceptable rendition. In Table 1 an example of emoji representing basic feelings is given.

As an example of the deployment of pictographs in sentiment expression, in January 2013, Facebook allowed users to post what they were feeling, watching, reading, drinking, eating, and more. Using a drop down menu, Facebook allowed users to express related feelings through a sentiment list containing many elements, such as happy, excited, loved, sad, crazy, blessed, perplexed, amused, hopeful, and so on.[7] This functionality has been limited to update status and is not applicable for comments or shared links. Furthermore, some Italian online newspapers (such "Corriere della Sera" and "Il Secolo XIX") and opinion blogs use software that measure audience sentiment. Commercial products such Vicomi allow readers to comment multimedia content with one type of reaction such "funny", "worried" or "angry".[8] This manual selection is heterogeneous for classes and their associated icons.

Unfortunately, what is gained in depth and information retrieval is lost in terms of simplicity, and the latter systems have not gained the universal acceptance that liking has. Choosing among different pictograms is no more a strictly atomic process, and requires direct action and a modicum of thinking, which makes it transforming into an habit a more difficult endeavour. While liking has become a sort of automatic reflex, sentiment expression still has to really catch on, as it has been hampered by less intuitive interfaces, as well as users' general laziness and lack of concentration or interest [9].

\subsection{Our approach to sentiment expression}

In this paper we outline a sentiment-expression hybrid system based on textual analysis [10] and linguistic fuzzy Markov chains [11], devised in order to overcome the intrinsic limitations of liking without burdening the user with complex choices. Some salient characteristics of the system are listed in the following.

- As sentiment expression is naturally done using linguistic items, often coupled by quantifiers (such as "a little sad", "very upset" and so on), the system has to natively deal with input and output formatted as such. This is obtained through the use of linguistic fuzzy Markov chains and of fuzzy linguistic operators [12].

- The system should avoid the complications brought by drop down menus, multiple icons and other complex interface elements, and should be inspired by the simplicity of liking. As such it should be able to predict a very limited number of feelings (and ideally just one), depending on previous interactions between the user expressing the sentiment and the original poster, and display the corresponding emoji for user interaction.

- In this context we will limit the scope of our system to sentiment expression about textual posts. Modularity of the system assures that an extension to other kinds of artifacts is possible by modifying only the first stage with the appropriate analysis algorithms.

As a more general comment, some of the authors of this paper have long debated and promoted the theory heralded by Enric Trillas [13] about the necessity of considering Fuzzy Set Theory more as an experimental science and how Cognitive Sciences are a perfect example of a discipline that should be empowered by interaction with FST $[14,15,16,17,18]$. The application we have outlined in this contribution aims to be a demonstration of how a more ecological approach to fuzzy problem solving in cognition can lead to efficient algorithms that are more relevant to the cognition problem itself, and does not force a solution onto a problem, but instead get shaped by it and adapt to naturally changing conditions.

\section{The Model}

The provisional system proposed in this paper consists of two stages: a fuzzy linguistic textual sentiment analysis, and a linguistic fuzzy Markov chain. The input of the system comes from posts on a SN or blog. The output is a linguistically graded emotion (eg. "Very Happy", "Mildly Angered"), represented by an emoji and a verbal label.

The first part provides a sort of ground truth for the textual content that the user should express a sentiment about. As outlined above, the first stage is obviously strictly dependent on the nature of the artifact. Judging the feelings an image should elicit is clearly different from the same task applied to a text or video, 
but the modular nature of the system should be considered a plus: a clear partition between ground truth and representation of users' sentiment allows customisation to different types of artifacts - providing suitable algorithms exist.

\subsection{Fuzzy linguistic textual sentiment analysis}

Sentiment analysis is a set of techniques through which it is possible to find, in automatic way, the general sentiment (or the opinion) expressed within a text. In particular sentiment analysis methodologies allow to find the polarity of the text to which are applied. Given an input text, sentiment analysis methodologies provide as output its polarity, which could be thought as a class label. In fact, the problems of sentiment analysis are a specific instance of classification problems, solvable through machine learning algorithms[19].

The sentiment analysis is employable in various application fields. In marketing, for example when a new product is launched in commerce; the producers, analyzing the opinion of the users, can understand the popularity of it, if it is or not appreciated by the users and why. Moreover, sentiment analysis can be important during the elections; is possible to find if an elector prefer one candidate over another through the opinions that they express, and so on.

The term opinion mining and sentiment analysis are often interchangeably and used with the same meaning but, as said in [20], "the concepts of emotions and opinions are not equivalent although they have a large intersection". It is clear that the emotional state of a person affect his opinion, but they are not the same things. So we can say that opinion mining methodologies are used to recognize the opinion polarity expressed in a text; instead the sentiment analysis methodologies try to guess the emotional state of the writer. In this work we focus our attention to the second set of methodologies to analyze a text.

There are different technique in literature to approach to sentiment analysis, each one with their peculiarity that tend to fit well with a particular problem within a specific application. The applying a combination of fuzzy logic and text analysis is not a new approach for discovering the right sentiment in a text. Different work in literature apply this type of techniques together to obtain a more accurate result. For example, in [21] the authors present a system for the multi-domain sentiment analysis to take in account the different polarity of each term respect to domain in which it is. to do this they use fuzzy logic theory for modelling of the membership function to relate concepts and domains. Or the work [22] where the authors try to consider the affective state through affective space.

\subsection{Linguistic fuzzy Markov chains}

There are two main approaches relative to fuzzy Markov chains. The most extended one use a fuzzy relation over the cartesian product between the state space. In literature this approach are used in application for speech recognition and image classification. [23] Buckley proposed a transition matrix as composed of fuzzy numbers, where domain for a given is:

$$
\operatorname{Dom}(\alpha)=\times_{i=1}^{n} \operatorname{Dom}_{i}(\alpha)
$$

$$
\begin{aligned}
\operatorname{Dom}(\alpha) & =\left(\times_{j=1}^{n} p_{i j}[\alpha]\right) \cap \Delta n= \\
& =\left(p_{i 1}, \cdots, p_{i n}\right) \in \mathbb{R}^{n}: \\
& p_{i j} \in\left[p_{i j}^{L}, p_{i j}^{U}\right] \wedge \sum_{j} p_{i j}=1
\end{aligned}
$$

He also defined a restricted fuzzy matrix multiplication (Eq. 1 and 2 ), that assures the probability restriction holds at the $\alpha$-cut level when doing computations with the transition matrix $P$ [11].

$$
\begin{array}{r}
P[\alpha]=\left(p_{i j}[\alpha]\right) \\
(P[\alpha])^{2}=\left(w_{i j}[\alpha]\right)=\sum_{k=1}^{r}\left(p_{i k}[\alpha] p_{k j}[\alpha]\right)
\end{array}
$$

In a recent implementation of linguistic fuzzy Markov chains, Villacorta, Verdegay and Pelta [23] suggest to address the uncertainty of linguist judgements by introducing fuzzy probabilities as an additional layer that is placed on top of Buckley's fuzzy Markov chains. Given a discrete linguistic probability distribution, $\pi_{1}, \cdots, \pi_{n}$, their sum must contain the singleton fuzzy number $1_{\chi}$, regardless of the type of fuzzy numbers involved or how the sum has been defined. For every row there is the constraint:

$$
p_{i 1}+\cdots+p_{i n} \supseteq 1_{\chi}, \forall i=1, \cdots, n
$$

If we consider Trapezoidal Fuzzy Numbers $A=$ $\left(a_{1}, b_{1}, c_{1}, d_{1}\right)$ and $B=\left(a_{2}, b_{2}, c_{2}, d_{2}\right)$, the sum is defined as follow:

$$
A+B=\left(a_{1}+a_{2}, b_{1}+b_{2}, c_{1}+c_{2}, d_{1}+d_{2}\right) .
$$

\section{Implementation}

In our implementation we consider "feelings" as fuzzy concept because a piece of content can be have different associated emotions. In fact there are hundred emotions that the human can feel and there are thousand way to express them into a text. Among the researchers there is still not a set of agreed basic emotions. However Ekman and Friesen list six basic emotions (happiness, anger, disgust, fear, sadness, surprise) [24]. They are not the only one to list basic emotions, but also [25] list another six primary emotions (love, joy, surprise, anger, sadness, fear). Also he assert that this primary emotion is not mutual exclusive when associated to an opinion, but they may have a different strength relative to the same opinion. In this work we consider the six basic emotions list from Ekman, but with the assertion 
that they have a strength and are not mutual exclusive. A user can be "very happy" and "a bit surprised" or "quite angry" and "mildly sad" when publish or read a status on a social network. For analyze this class of texts, we use a classical approach to quantify the strength of each feeling, followed by an interaction prediction phase that correlate the possible range of emotions with the previous interaction between two users. These information from the two phases can be used for calculate in a hybrid fuzzy system the appropriated emoji.

In this preliminary work we use probabilistic tools such machine learning algorithm and stochastic process, because these techniques are well-assessed in literature, in particular in the field of NLP. In the following sections we use alternatively the terms "feelings", "emotions", "sentiments" and "emoji" considering a bijective map from one set to the other.

From this point onwards we consider two actors: we call $\mathrm{A}$ the actor that writes the content that the actor B will express liking.

\subsection{Text analysis}

The first step of our method consist of the analysis of the text to recognise the sentiment orientation that his author A express. As we said in before this is a very hard task for a non-human reader. In order to simplify the problem we decided to take in account only six sentiments [24], because these emotions are universally recognized and have different typical expressions. For an human reader it is quite easy, for the most of written content, recognise the main class associated with a text and likewise is easy classify a text in one of these classes using some of the major techniques of text mining. But this approach mask all the possible emotions present in text with different weights.

There are many different algorithms of text mining for the sentiment classification, each of this can have a different output. As said before the most commons output for classification algorithms are a single class, for example the decision tree [26] provides in output only the more probable class which the text agrees. Instead other algorithms like a Bayesian, Support Vector Machines (SVM) or nearest-neighbor (NN) classifier also provide the membership degree (as distance or probability).

For this step we use the text analysis to discover the objective opinion expressed by the content shared by A, used in the third step for the prediction of correct feeling of the user B. In our work we consider the Google Prediction Framework. This framework allows to train a model with a training set, containing social network contents random gathered among public profiles or friend's profiles, previously manually labelled by users with one of the possible emotion labels. This procedure has a slight weakness: as mentioned before, for a human reader discover the main emotion in a text is an easy task, but he can also recognize the others minor emotions that may be present in the text. This behaviour cannot be represented in our model of training-set, but with the following phases it is possible to consider this aspect. After the training phase, the model can be used to predict the label of a new data, that have to be of the same type of the training set data. The output of the model consist in a probabilistic weight vector, where the position $p_{i}$ is the probability that the data belong to the class $p_{i}$. This weight can be interpreted as membership degrees of feeling $i$ present in the text written by A.

\subsection{Interaction prediction}

The second step takes advantages of the fact that a social network site is a bounded system, in which users have connections to each others [27]. These information can be used to adjust a general analysis based only of the content and exploit the history between users that occurs during a period of time.

In this prediction phase we evaluate previous interactions between two individuals using the linguistic fuzzy Markov chain. The state space is the set of selected emoji with a membership degree for each feeling. The updating transition matrix can be used to predict the next emoji used from the user B to express is feeling toward a generic piece of content shared by the user $\mathrm{A}$.

In our model we implement a similar approach to the one used by Villacorta et al. [23], using five possible linguistic labels (see Figure 2) to evaluate the probability that the chain moves from one state to another. For the sake of simplification, trapezoidal membership functions (such as the ones suggested by Bonissone [12]) can be used, and the number of linguistic terms can also be adapted at will.

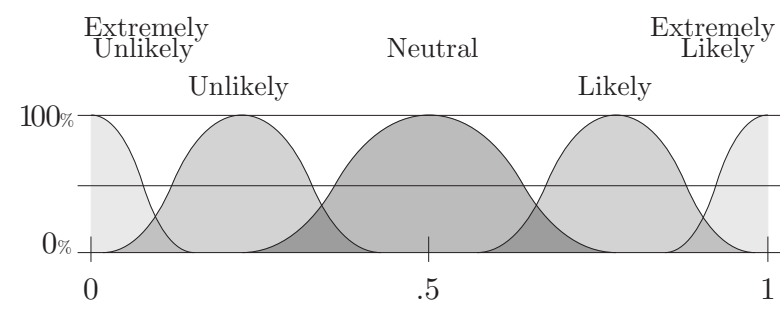

Figure 2: Membership functions of linguistic probability labels for transitions.

Both the vector state and the prediction matrix, are firstly set with uniform values, indicating that a base condition where the user $\mathrm{B}$ does not express any feeling towards the posts of $\mathrm{A}$, so the next interaction between the two users is not affected by previous interactions, but only by the evaluation given by text analysis about the content written by A.

After each interaction in which the user B selects the suggested emoji, we update the state vector to reflect his alteration of feelings, boosting the emotion selected from B and decreasing the others emoji. We also take in account the row used for the prediction, updating the weights according to the choice of $\mathrm{B}$, increasing the entry of the new selected feeling and decreasing the remaining elements. This reinforces the effect of selection for future predictions. This procedure strengthens the previous choices, making the weights associated with 
emoji more personal and linked with the relationship between the two actors of communication.

The output of this phase is a set of emoji, sorted by overall weight, with the membership degree converted in a description expressed with the natural language (using adjectives such "very", "quite", etc.). The better result can be extracted and depicted as in Figure 3. A caption can explain the meaning of the suggested emoji, likewise the other button short description ("Like" for thumbs up sign, "Share" for arrow pointing upwards then curving rightwards). The social network user can select the proposed emoji (in a direct liking-like way) or open a drop down menu that contains other options, sorted by weight with their associated description.

The selection of any of the possible emojis acknowledge the author of the post about the feeling of his contact. The interface of the button and the notification method can vary according the SN considered and the personal settings of the users.

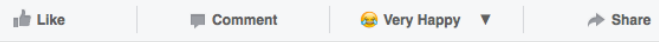

Figure 3: Example of a "Very Happy" button ("face with tears of joy" emoji), chosen by the system, as it would be displayed in the Facebook interface.

\section{Conclusions and further research}

First tests of a sketched implementation of the system are consistent with the experimental hypothesis. The algorithm seems able to select a single linguistically graded feeling related to the text itself and the feeling of the user toward the original poster.

As with any text-analysis based approach, our system suffers from possible misinterpretation of comment that contains sarcasm and conflicting sentiment (e.g. an apparent sad message that conceal an humorous meaning) [28]. This is a direct consequences of the process of text evaluation in the first phase, and can be mitigated by the development of a smarter and more nuanced text sentiment analysis engine.

Another possible shortcoming of the way the system has been devised is due to the fact that sentiments about an artifact have further reasons and motives than the ones derived by previous interactions at a social network level: general attitudes, facts of life and personal dispositions are often of a greater influence on feelings than what happens online. As more and more interactions are mirrored by online artifact, this kind of problem should be alleviated. We contend that, albeit the kind of system proposed would never achieve a rate of prediction close to perfection, the improvement guaranteed by it would nonetheless accelerate the adoption of sentiment expression add-ons to the existing liking interfaces.

In a possible implementation of the system in a simple social network (such as one based on open source framework Elgg [29]), the selection of an emoji can be used to improve both text analysis and interaction prediction providing a test and training phase could be devised. The system would obviously benefit from a medium scale training phase, in order to calibrate the updating function of the fuzzy Markov chain, and to experimentally test the theoretical hypothesis of an increased use of sentiment validation by the user when a simpler interface is presented. Unfortunately such kind of tests would require sizeable resources, and to be representative of real world usage it would benefit from the collaboration with an established SN.

It has to be stressed the fact that the system outlined in this paper is based thoroughly on linguistic descriptions, and that in any phase of the algorithm linguistic representations, under the guise of fuzzy numbers, are employed. No external model is forced on the system or the user: any step of the text analysis and of the Markov chain updating is done in what we maintain is a more ecological, transparent way. Any partial analysis of the system will reveal is inner workings in terms of sentiments and their relative strength, an the evolution of the system is clearly readable and understandable, even with the help of visualization.

As for future development, the system can be implemented with different improvements and modification to better tailor a wide range of applications: different modules for different kinds of artifacts should be implemented, with a special care for moving and still images, whose baseline evaluation is still far to be solved in an efficient way; links and hashtags could be better pre-analyzed for a better prediction from general trends when content which can be considered the same or similar is shared by different users.

\section{Acknowledgements}

This work has been supported by P.O. F.E.S.R Sicilia 2007-2013 Progetto POSING - Investiamo nel vostro futuro - l'Europa cresce in Sicilia.

\section{References}

[1] Charles Steinfield, Nicole B Ellison, and Cliff Lampe. Social capital, self-esteem, and use of online social network sites: A longitudinal analysis. Journal of Applied Developmental Psychology, 29(6):434-445, 2008.

[2] Keith Hampton, Lauren Sessions Goulet, Lee Rainie, and Kristen Purcell. Social networking sites and our lives. 2011.

[3] D. Lee. Facebook thinking about 'dislike' function - Zuckerberg, Available online 2014.

[4] M. Davis and P. Edberg. Proposed draft unicode technical report \#51, Available online 2014.

[5] A. Sternbergh. Smile, you're speaking emoji, Available online 2014.

[6] Harald G Wallbott and Klaus R Scherer. Cues and channels in emotion recognition. Journal of personality and social psychology, 51(4):690, 1986.

[7] J. Constine. Facebook asks you to please select your emotion, Available online 2013. 
[8] Eli Ken-Dror. System and method for organizing and designing comment, January 8 2014. US Patent App. 14/149,904.

[9] Wenhong Chen and Kye-Hyoung Lee. Sharing, liking, commenting, and distressed? the pathway between facebook interaction and psychological distress. Cyberpsychology, Behavior, and Social Networking, 16(10):728-734, 2015/01/30 2013.

[10] Alan McKee. Textual analysis: A beginner's guide. Sage, 2003.

[11] James J Buckley and Esfandiar Eslami. Fuzzy Markov chains: uncertain probabilities. Mathware \& soft computing, 9(1):33-41, 2008.

[12] Piero P Bonissone and Keith S Decker. Selecting uncertainty calculi and granularity: An experiment in trading-off precision and complexity. Proc. of the Conf. Annual Conference on Uncertainty in Artificial Intelligence (UAI-85), 2013.

[13] Enric Trillas and Sergio Guadarrama. Fuzzy representations need a careful design. International Journal of General Systems, 39(3):329-346, 2010.

[14] Marco Elio Tabacchi and Settimo Termini. Some reflections on fuzzy set theory as an experimental science. In Anne Laurent, Olivier Strauss, Bernadette Bouchon-Meunier, and Ronald R. Yager, editors, Information Processing and Management of Uncertainty in Knowledge-Based Systems, volume 442 of Communications in Computer and Information Science, pages 546-555. Springer International Publishing, 2014.

[15] Settimo Termini and Marco Elio Tabacchi. Fuzzy set theory as a methodological bridge between hard science and humanities. International Journal of Intelligent Systems, 29(1):104-117, 2014.

[16] Rudolf Seising and Marco Elio Tabacchi. The webbed emergence of fuzzy sets and computer science education from electrical engineering. In Davide Ciucci, Javier Montero, and Gabriella Pasi, editors, Proceedings of the 8th conference of the European Society for Fuzzy Logic and Technology, Advances in Intelligent Systems Research. European Society for Fuzzy Logic and Technology, Atlantis Press, 2013.

[17] Marco Elio Tabacchi and Settimo Termini. Theory of computation, fuzziness and a physics of the immaterial. In Davide Ciucci, Javier Montero, and Gabriella Pasi, editors, Proceedings of the 8th conference of the European Society for Fuzzy Logic and Technology, Advances in Intelligent Systems Research. European Society for Fuzzy Logic and Technology, Atlantis Press, 2013.

[18] Marco Elio Tabacchi and Settimo Termini. A few remarks on the roots of fuzziness measures. In Salvatore Greco, Bernadette Bouchon-Meunier, Giulianella Coletti, Mario Fedrizzi, Benedetto Matarazzo, and RonaldR. Yager, editors, $A d$ vances in Computational Intelligence, volume 298 of Communications in Computer and Information Science, pages 62-67. Springer Berlin Heidelberg, 2012.
[19] A. Zadgaonkar A. Buche, Dr. M. B. Chandak. Opinion mining and analysis: A survey. IJNLC, 2(3), june 2013.

[20] Bing Liu. Sentiment analysis and subjectivity. Handbook of natural language processing, 2:627666, 2010.

[21] Mauro Dragoni, Andrea GB Tettamanzi, and Célia da Costa Pereira. A fuzzy system for concept-level sentiment analysis. In Semantic Web Evaluation Challenge, pages 21-27. Springer, 2014.

[22] Fabon Dzogang, M-J Lesot, Maria Rifqi, and Bernadette Bouchon-Meunier. Expressions of graduality for sentiments analysis? a survey. In Fuzzy Systems (FUZZ), 2010 IEEE International Conference on, pages 1-7. IEEE, 2010.

[23] Pablo Villacorta, Jose Luis Verdegay, and David Pelta. Towards fuzzy linguistic Markov chains. In 8th conference of the European Society for Fuzzy Logic and Technology (EUSFLAT-13). Atlantis Press, 2013.

[24] Paul Ekman and Wallace V Friesen. Constants across cultures in the face and emotion. Journal of personality and social psychology, 17(2):124, 1971.

[25] W Gerrod Parrott. Emotions in social psychology: Essential readings. Psychology Press, 2001.

[26] Bing Liu. Web data mining: exploring hyperlinks, contents, and usage data. Springer Science \& Business Media, 2007.

[27] Nicole B Ellison et al. Social network sites: Definition, history, and scholarship. Journal of Computer-Mediated Communication, 13(1):210230, 2007.

[28] Roberto González-Ibánez, Smaranda Muresan, and Nina Wacholder. Identifying sarcasm in Twitter: a closer look. In Proceedings of the 49th Annual Meeting of the Association for Computational Linguistics: Human Language Technologies: short papers-Volume 2, pages 581-586. Association for Computational Linguistics, 2011.

[29] M. Sharma. Elgg Social Networking. From Technologies to Solutions. Packt Publishing, Limited, 2008. 